\title{
FDG PET/CT - not PSMA trendy, but available, comfortable, and complementary
}

\section{Robert J. Hamilton, MD, MPH, FRCSC}

Princess Margaret Cancer Centre, Toronto, ON, Canada

Cite as: Hamilton RJ. FDG PET/CT - not PSMA trendy, but available, comfortable, and complementary. Can Urol Assoc J 2021;15(10):308-9. http://dx.doi.org/10.5489/cuaj.7595

\section{See related article on page 301}

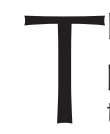
here is no doubt we are amidst an imaging revolution in prostate cancer. Attend any oncology-based meeting in the last five years and "next-generation" positron emission tomography (PET) imaging is discussed as if as common as prostate-specific antigen (PSA). Talk to our colleagues in Australia, Europe, and the U.S., and we get the same feeling. For those in Canada at the handful of institutions where prostate-specific membrane antigen (PSMA) PET is being studied, we have felt that next-generation imaging has already been integrated into general practice. The reality though, is that for the average Canadian with prostate cancer, next-generation imaging is not readily available. Moreover, there is a dearth of high-quality data supporting that next-generation imaging translates into improved prostate cancer outcomes.

So, while we drool with anticipation of approval and funding of novel PET tracers, it makes sense to study one of the oldest and most commonly used tracers in oncology ${ }^{18} \mathrm{~F}$-fluorodeoxyglucose $\left({ }^{18} \mathrm{~F}-\mathrm{FDG}\right)$.

In this edition of CUAJ, Otis-Chapados et al performed a retrospective study of 256 patients undergoing primary staging for high-grade (International Society of Urological Pathology [ISUP] $\geq 4$ ) prostate cancer. ${ }^{1}$ All patients underwent a bone scan and an FDG PET/computed tomography $(\mathrm{CT})$. They analyzed the imaging results as a function of the "standard of truth." defined as confirmation/development of definitive bone metastases with either a bone biopsy, new or confirmed metastasis on followup conventional imaging, or clinical followup compatible with bone metastasis. They observed that FDG-PET/CT detected more metastases than bone scans with superior sensitivity $(100 \%$ vs. $78.8 \%)$ and specificity (98.7\% vs. 98.2\%). They concluded that FDGPET/CT should be used as the sole imaging modality for prostate cancer staging.

The finding of FDG-PET superiority is perhaps surprising to some. Traditional thinking was that, unlike other genitouri- nary cancers, such as urothelial, penile, and testis, prostate cancers less frequently adopt glycolysis as their preferred energy production mechanism, and this explains why some studies showed a lack of utility for FDG-PET. ${ }^{2}$ While true of primary, especially low-grade prostate cancers, more highgrade, metastatic, castration-resistant or neuroendocrine differentiated prostate cancers increasingly use glycolytic metabolism. ${ }^{3}$ Thus, it is plausible that in this cohort of highgrade prostate cancers, FDG-PET would perform well.

At first glance, one may think there is little merit in pursuing FDG-PET as standard of care imaging when much more sensitive tracers are being actively studied. For example, PSMA-based tracers lead the pack, with more impressive performance in both primary staging and recurrent settings. ${ }^{4,5}$ The recent ProPSMA randomized control trial demonstrated PSMA-PET to be vastly superior to conventional imaging in primary staging. ${ }^{6}$ Moreover, the field of PSMA-based imaging is evolving rapidly. Even more sensitive PSMA-based tracers are being evaluated (e.g., PSMA-1007) (NCT04644822) and a Canadian-led, multicenter, prospective trial (PATRON) is underway to evaluate whether using PSMA-PET/CT can translate into improved outcomes (NCT04557501).

However, the findings of Otis-Chapados et al shouldn't be disregarded. The group at Université Laval and others have taught us that FDG avidity gives insight into the biology of prostate cancer. FDG-avidity correlates with GLUT1 expression, adverse pathology, nodal involvement, and worse outcomes. ${ }^{3,7}$ While PSMA-PET is more sensitive than FDG-PET in general, there are clearly prostate cancers that do not express PSMA but avidly uptake FDG. ${ }^{8}$ Even within the same patient, there may be heterogeneity among metastatic sites, with some more PSMA-avid and others more FDG-avid, and this heterogeneity can provide insight into how those metastases may behave with certain treatments. ${ }^{9}$ Studies have even shown concomitant use of PSMA and FDG not only increases metastasis yield but helps better characterize the disease. ${ }^{10}$

So, as we await PSMA approval, as we scramble for even more sensitive PSMA tracers, and as we face the reality that we don't actually know what to do with finding such tiny amounts of cancer in this imaging revolution, maybe Otis- 
Chapados et al are showing us an alternative pathway with FDG-PET — available, comfortable, and complementary.

Competing interests: Dr. Hamilton is an advisory board member for Astellas, Bayer, Janssen, and TerSera; and has participated in clinical trials supported by Astellas, Bayer, and Janssen.

\section{References}

1. Otis-Chapados S, Ringuette Goulet C, Dubois G, et al. ${ }^{18 F-f l u o r o d e o x y g l u c o s e ~ p o s i t r o n ~ e m i s s i o n ~ t o m o g r a-~}$ phy/computed tomography (PET/CT) is accurate for high-grade prostate cancer bone staging when compared to bone scintigraphy. Can Urol Assoc J 2021;15:301-7. http://dx.doi.org/10.5489/cuaj.7107

2. Jadvar H. Imaging evaluation of prostate cancer with 18F-fluorodeoxyglucose PET/CT: Utility and limitations. Eur J Nucl Med Mol / 2013;40:5-10. https://doi.org/10.1007/s00259-013-2361-7

3. Beauregard J-M, Blouin A-C, Fradet V, et al. FDG-PET/CT for pre-operative staging and prognostic stratification of patients with high-grade prostate cancer at biopsy. Cancer Imag 2015;15:2. https://doi. org/10.1186/s40644-015-0038-0

4. Fourquet A, Rosenberg A, Mena E, et al. A comparison of ${ }^{18}$-DCFPyL, ${ }^{18} \mathrm{~F}-\mathrm{NaF}$, and ${ }^{18} \mathrm{~F}-\mathrm{FDG} \mathrm{PET} / \mathrm{CT}$ in a prospective cohort of men with metastatic prostate cancer. J Nucl Med 2021; Epub ahead of print. https://doi.org/10.2967/inumed.121.262371

5. Jadvar H, Desai B, Ji L, et al. Prospective evaluation of ${ }^{18 F-N a F}$ and ${ }^{18 F}$-FDG PET/CT in detection of occult metastatic disease in biochemical recurrence of prostate cancer. Clin Nucl Med 2012;37:637-43. https:// doi.org/10.1097/RLU.0b013e318252d829
6. Hofman MS, Lawrentschuk N, Francis RJ, et al. Prostate-specific membrane antigen PET-CT in patients with high-risk prostate cancer before curative-intent surgery or radiotherapy (proPSMA): A prospective, randomized, multicenter study. Lancet 2020; 395:1208-16. https://doi.org/10.1016/S01406736(20)30314-7

7. Meziou S, Goulet CR, Hovington H, et al. GLUTl expression in high-risk prostate cancer: correlation with ${ }^{18 F-F D G-P E T / C T ~ a n d ~ c l i n i c a l ~ o u t c o m e . ~ P r o s t a t e ~ C a n c e r ~ P ~ D ~ 2020 ; 23: 441-8 . ~ h i t p s: / / d o i . o r g / 10.1038 / ~}$ s41391-020-0202-x

8. Bakht MK, Lovnicki JM, Tubman J, et al. Differential expression of glucose transporters and hexokinases in prostate cancer with a neuroendocrine gene signature: A mechanistic perspective for ${ }^{18} \mathrm{~F}-\mathrm{FDG}$ imaging of PSMA-suppressed tumors. J Nucl Med 2020;61:904-10. https://doi.org/10.2967/inumed.119.231068

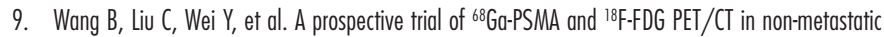
prostate cancer patients with an early PSA progression during castration. Clin Cancer Res 2020;26:4551-8. https://doi.org/10.1158/1078-0432.CCR-20-0587

10. McGeorge $S$, Kwok M, Jiang A, et al. Dual-tracer positron-emission tomography using prostate-specific membrane antigen and fluorodeoxyglucose for staging of prostate cancer: A systematic review. Adv Urology 2021;2021:1-9. htrps://doi.org/10.1155/2021/1544208

Correspondence: Dr. Robert J. Hamilton, Princess Margaret Cancer Centre, Toronto, ON, Canada; Rob.Hamilton@uhn.co

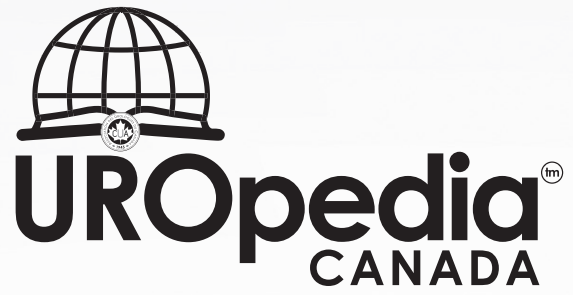

\section{Your one stop knowledge centre}

Learn and earn credits by visiting CUA's educational library. New content is added weekly!

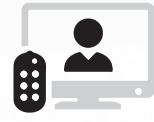

\section{Webinar Recordings}

- CUA Highlights of ESMO 2021

- Thomas Powles: Update on the Treatment Landscape for Urothelial Carcinoma in Canada

- Updated Guidance for Hereditary Testing for Prostate Cancer in Ontario

\section{Virtual Meetings}

- PMCC Biomarker Bootcamp

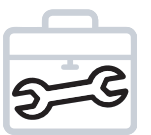

\section{Clinical Toolkits to Improve Your Practice}

- Advanced Prostate Cancer: Treat/Refer Guide

- Advanced Renal Cell Carcinoma: Patient Guide and Diary (includes pembrolizumab+axitinib \& ipilimumab+nivolumab treatment diaries)

\section{cua.org/UROpedia}

